\title{
Discrete Mukherjee-Islam Distribution and Its Characterization
}

\author{
Sabir Ali Siddiqui ${ }^{a}$, Sanjay Jai ${ }^{\mathrm{b}}$, Husam EdlinSadig Ahmed Sadig ${ }^{\mathrm{c}}$, Shradha Dwivedi ${ }^{\mathrm{d}}$, Raed Abdel kareem \\ a,e Department of Mathematics and Sciences, Dhofar University, Salalah, Sultanate of Oman. \\ ${ }^{b}$ Department of Statistics, St. John's College, Agra, Uttar Pradesh (India). \\ ${ }^{\mathbf{c}}$ Department of Mathematics and Sciences, Dhofar University, Salalah, Sultanate of Oman \\ ${ }^{\mathrm{d}}$ Mathematics, IT Unit, Salalah College of Technology, Sultanate of Oman \\ Email: \\ asabir_siddiqui@du.edu.om,,drsanjayjain.sjc@gmail.com, ${ }^{\mathrm{c}}$ hsadig@du.edu.om, ${ }^{\mathrm{d}}$ shradha.d@ @st.edu.om, raed@du.edu.om
}

Article History: Received: 10 November 2020; Revised 12 January 2021 Accepted: 27 January 2021; Published online: 5 April 2021

\begin{abstract}
In the present paper an attempt has been made to introduce discrete form of a well-known and widely used continuous distribution Mukherjee-Islam (MI) distribution. Further some characteristics of the discrete MI distribution have been discussed along with the estimation of its parameters.
\end{abstract}

Keywords: Mukherjee-Islam distribution, Continuous and Discrete distribution, Discretization

\section{Introduction}

In most of the studies of reliability analysis, survival analysis, fertility analysis etc., it is assumed that the variable under study is continuous in nature and therefore continuous distribution were used to characterize the phenomenon under study. But there are many situations where this assumption may not be fit well and we prefer the application of a continuous distribution due to mathematical convenience and also due to nonavailability of discrete distribution for the undergoing study.

There are many real-life situations where discrete distributions are more helpful than the continuous distribution, discrete distributions have a great importance in health statistics, in business and in inventory control.

In last years a number of researchers worked and introduced some new discrete distributions. Jayakumar and Girish Babu (2017) studies discrete Weibull geometric distribution and its properties.

In the present paper an attempt has been made to develop a discrete form of continuous Mukherjee-Islam distribution by discretizing the cumulative distribution function (cdf) of MI distribution as discussed in Chakraborty (2015).

\section{Mukherjee-Islam Distriution}

In 1983, Mukherjee and Islam discussed the properties of a finite-range failure-time distribution, that includes the exponential as a particular case. The distribution was IFR when the shape parameter exceeds unity, but is IFRA always. Jain (2015) used this distribution in fertility analysis to study the waiting time for first conception. Siddiqui et al. (2016) introduced beta exponentiated Mukherjee-Islam distribution. Rather and Subramanian (2018) studied the family of distributions termed as exponentiated Mukherjee-Islam distribution. Dar et al. (2018) derived a new weighted class Mukherjee-Islam distribution by taking the distribution function as a weight. The pdf of Mukherjee-Islam distribution is

$$
\begin{aligned}
& \mathrm{f}(\mathrm{x})=\left(\frac{p}{x}\right)\left[\left(\frac{x}{\theta}\right)^{p}\right] \quad ; \quad \mathrm{p}, \theta>0 ; 0<\mathrm{x} \leq \theta \\
& =0 \quad \text { otherwise }
\end{aligned}
$$

Where $\theta$ and $\mathrm{p}$ are the scale and shape parameters respectively. The model is monotonic decreasing and highly skewed to the right. The graph is J-shaped thereby showing the unimodal feature.

The cumulative distribution function $\mathrm{F}(\mathrm{x})$, reliability function $\mathrm{R}(\mathrm{x})$ and hazard rate function for $\mathrm{MI}$ distribution are 


$$
\begin{array}{ll}
\mathrm{F}(\mathrm{x})=\left(\frac{x}{\theta}\right)^{p} ; & \mathrm{p}, \theta>0 ; 0<\mathrm{x} \leq \theta \\
\mathrm{R}(\mathrm{x})=1-\left(\frac{x}{\theta}\right)^{p} ; & \mathrm{p}, \theta>0 ; 0<\mathrm{x} \leq \theta \\
\mathrm{h}(\mathrm{x})=\frac{p x^{p-1}}{\left(\theta^{p}-x^{p}\right)} ; & \mathrm{p}, \theta>0 ; 0<\mathrm{x} \leq \theta
\end{array}
$$

The other salient features are

Mean

$$
\mathrm{E}(\mathrm{x})=\left(\frac{p}{p+1}\right) \cdot \theta
$$

Variance

$$
\mathrm{V}(\mathrm{x})=\frac{p}{(p+1)(p+2)} \cdot \boldsymbol{\theta}
$$

rth moment about Origin

$$
\mu_{r}^{\prime}=\left(\frac{p}{p+r}\right) \cdot \theta^{r}
$$

\section{Discrete Mukherjee-Islam Distriution}

By using the cdf and reliability function of the Continuous Mukherjee-Islam Distribution (CMID) we obtained the probability mass function (pmf) for Discrete Mukherjee-Islam Distribution (DMID) as follows:

$$
\begin{aligned}
& \mathrm{P}(\mathrm{Y}=\mathrm{y})=\mathrm{P}(\mathrm{X} \leq \mathrm{y}+1)-\mathrm{P}(\mathrm{X} \leq \mathrm{y}) \\
& \quad \mathrm{F}(\mathrm{y}+1)-\mathrm{F}(\mathrm{y})=\mathrm{R}(\mathrm{y})-\mathrm{R}(\mathrm{y}+1)=\left(\frac{y+1}{\theta}\right)^{p}-\left(\frac{y}{\theta}\right)^{p} \\
& \mathrm{P}(\mathrm{Y}=\mathrm{y})=\frac{(y+1)^{p}-(y)^{p}}{\theta^{p}} \quad ; \mathrm{y}=0,1,2, \ldots \ldots, \theta-1
\end{aligned}
$$

Summing the equation (5) over the range $y=0,12, \ldots ., \theta-1$,

$\sum_{y=0}^{\theta-1} \frac{(y+1)^{p}-(y)^{p}}{\theta^{p}}=1$.
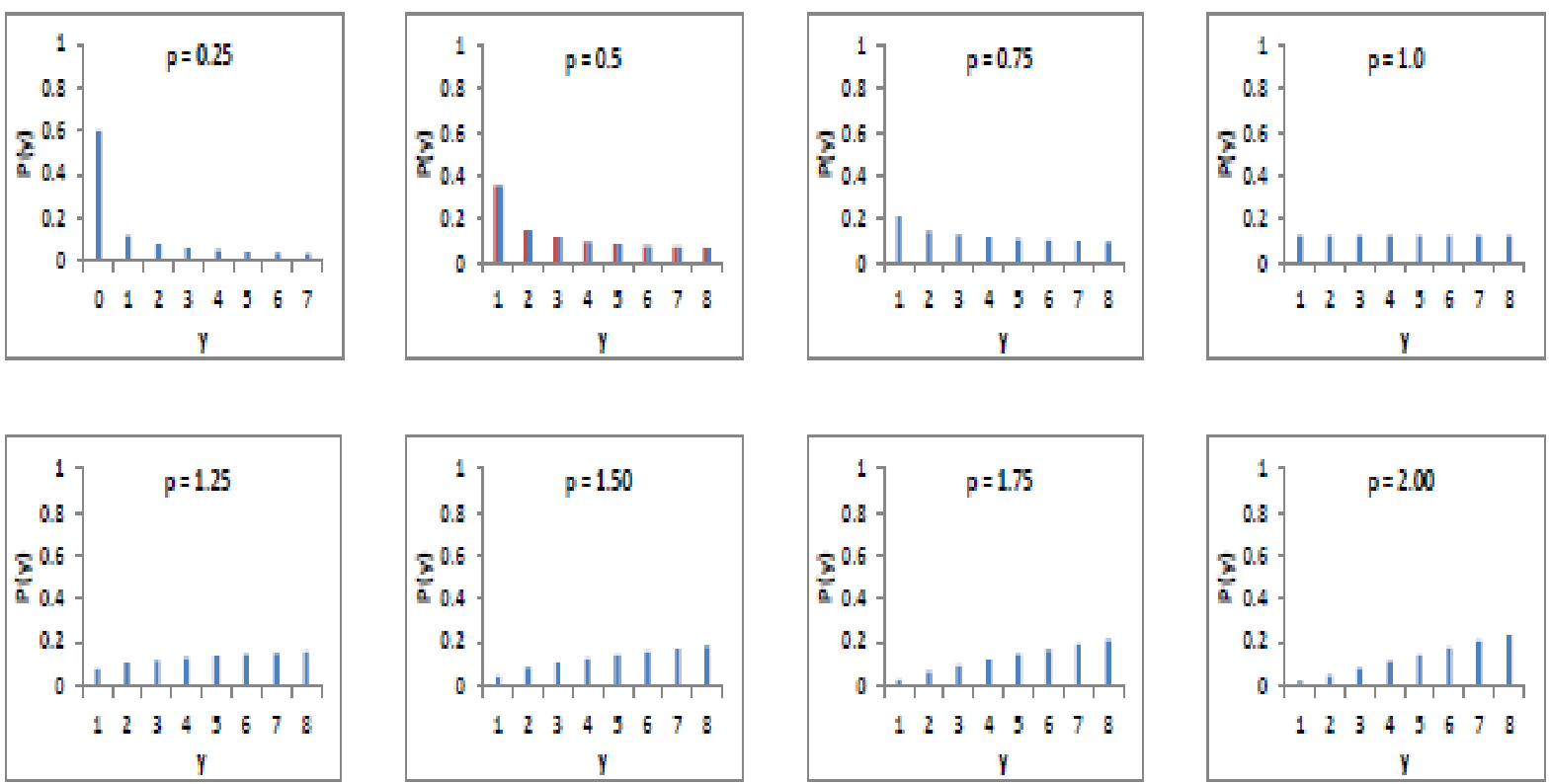

Figure 1: Plots of pmf of DMID for $\theta=8$ and different choices of $p$.

The cumulative distribution function $\mathrm{F}(\mathrm{x})$, for DMID will be

$$
\mathrm{F}(\mathrm{y})=\left(\frac{y+1}{\theta}\right)^{p} \quad ; \mathrm{y}=0,1,2, \ldots \ldots, \theta-1
$$

here, $\mathrm{F}(0)=\frac{1}{\theta^{p}} \mathrm{i}$.e the proportion of positive values, $1-\mathrm{F}(0)=\frac{\theta^{p}-1}{\theta^{p}}$

and also $\quad \mathrm{P}(\mathrm{a}<\mathrm{Y} \leq \mathrm{b})=\frac{(b+1)^{p}-(a+1)^{p}}{\theta^{p}}$

The other salient features are 
Mean:

$$
\begin{aligned}
& \mathrm{E}(\mathrm{x})=(\theta-1)-\sum_{k=1}^{\theta-1}\left(\frac{k}{\theta}\right)^{p} \\
= & (\theta-1)-\frac{1}{\theta^{p}} \mathrm{H}_{\theta-1}^{(-p)} ;
\end{aligned}
$$

where $H_{n}\left(=\sum_{k=1}^{n} \frac{1}{k}\right)$ is a harmonic number.

and

$$
\mathrm{E}(\mathrm{x} 2)=(\theta-1)^{2}-\sum_{k=1}^{\theta-1}(2 k-1)\left(\frac{k}{\theta}\right)^{p}
$$

Further, variance can be obtained by using following relation.

$\operatorname{Var}(\mathrm{x})=\mathrm{E}(\mathrm{x} 2)-[\mathrm{E}(\mathrm{x})] 2$

Table 1 : Values of Mean, Variance and Coefficient of Variance for $\theta=10$ and for different choices of $p$.

\begin{tabular}{|l|l|l|l|l|l|l|l|l|}
\hline & $\begin{array}{l}\mathrm{p}= \\
0.25\end{array}$ & $\begin{array}{l}\mathrm{p}=0.5 \\
0\end{array}$ & $\begin{array}{l}\mathrm{p}=0 . \\
75\end{array}$ & $\begin{array}{l}\mathrm{p}=1 . \\
00\end{array}$ & $\begin{array}{l}\mathrm{p}=1 . \\
25\end{array}$ & $\begin{array}{l}\mathrm{p}=1 . \\
50\end{array}$ & $\begin{array}{l}\mathrm{p}=1 . \\
75\end{array}$ & $\begin{array}{l}\mathrm{p}=2 . \\
00\end{array}$ \\
\hline $\mathrm{E}(\mathrm{X})$ & 1.678 & 2.895 & $\begin{array}{l}3.80 \\
3\end{array}$ & $\begin{array}{l}4.50 \\
0\end{array}$ & $\begin{array}{l}5.04 \\
8\end{array}$ & $\begin{array}{l}5.48 \\
8\end{array}$ & $\begin{array}{l}5.84 \\
9\end{array}$ & $\begin{array}{l}6.15 \\
0\end{array}$ \\
\hline $\mathrm{E}(\mathrm{X} 2)$ & 9.280 & 16.87 & 23.1 & 28.5 & 33.0 & 36.9 & 40.3 & 43.3 \\
& & 1 & 81 & 00 & 38 & 52 & 59 & 50 \\
\hline Var(X & 6.464 & 8.491 & 8.71 & 8.25 & 7.55 & 6.83 & 6.14 & 5.52 \\
) & & 7 & 0 & 7 & 0 & 5 & 8 \\
\hline C.V.( & 151.5 & 100.6 & 77.6 & 63.8 & 54.4 & 47.6 & 42.3 & 38.2 \\
\%) & 00 & 56 & 29 & 28 & 58 & 19 & 82 & 29 \\
\hline
\end{tabular}

The reliability function $\mathrm{R}(\mathrm{x})$ and hazard rate function for DMID will be

$\mathrm{R}(\mathrm{y})=\mathrm{P}(\mathrm{Y}>\mathrm{y})=1-\mathrm{P}(\mathrm{Y} \leq \mathrm{y})$

$=1-\left(\frac{y+1}{\theta}\right)^{p} ; \mathrm{y}=0,1,2, \ldots \ldots, \theta-1$

and

$\mathrm{h}(\mathrm{y})=\frac{P(Y=y)}{P(Y \geq y)}=\frac{(y+1)^{p}-(y)^{p}}{\theta^{p}-y^{p}} \quad ; \mathrm{y}=0,1,2, \ldots \ldots, \theta-1$

In particular, if $\mathrm{y}=\theta-1$,

$\mathrm{h}(\theta-1)=\frac{(\theta-1+1)^{p}-(\theta-1)^{p}}{\theta^{p}-(\theta-1)^{p}}=1$; representing that in a survival model $100 \%$ of the survived lives die off and if $\mathrm{y}$ $=0$,

$\mathrm{h}(0)=\frac{(1)^{p}-(0)^{p}}{\theta^{p}-(0)^{p}}=\frac{1}{\theta^{p}}=\mathrm{F}(0)$

Further, for $\mathrm{p}=1$

$\mathrm{h}(\mathrm{y})=\frac{(y+1)^{1}-(y)^{1}}{\theta^{p}-(y)^{1}}=\frac{1}{\theta^{p}-y}$

Table 3 : Values of hazard rate function for $\theta=10$ and for different choices of $p$.

\begin{tabular}{|c|l|l|l|l|l|l|l|l|}
\hline $\mathrm{Y}$ & $\begin{array}{l}\mathrm{p}= \\
0.25\end{array}$ & $\begin{array}{l}\mathrm{p}=0.5 \\
0\end{array}$ & $\begin{array}{l}\mathrm{p}=0.7 \\
5\end{array}$ & $\begin{array}{l}\mathrm{p}=1.0 \\
0\end{array}$ & $\begin{array}{l}\mathrm{p}=1.2 \\
5\end{array}$ & $\begin{array}{l}\mathrm{p}=1.5 \\
0\end{array}$ & $\begin{array}{l}\mathrm{p}=1.7 \\
5\end{array}$ & $\begin{array}{l}\mathrm{p}=2.0 \\
0\end{array}$ \\
\hline 0 & $\begin{array}{l}0.56 \\
2\end{array}$ & 0.316 & 0.178 & 0.100 & 0.056 & 0.032 & 0.018 & 0.010 \\
\hline 1 & $\begin{array}{l}0.24 \\
3\end{array}$ & 0.192 & 0.147 & 0.111 & 0.082 & 0.060 & 0.043 & 0.030 \\
\hline
\end{tabular}




\begin{tabular}{|c|l|l|l|l|l|l|l|l|}
\hline 2 & $\begin{array}{l}0.21 \\
5\end{array}$ & 0.182 & 0.152 & 0.125 & 0.102 & 0.082 & 0.066 & 0.052 \\
\hline 3 & $\begin{array}{l}0.21 \\
2\end{array}$ & 0.187 & 0.164 & 0.143 & 0.124 & 0.106 & 0.091 & 0.077 \\
\hline 4 & $\begin{array}{l}0.22 \\
3\end{array}$ & 0.203 & 0.184 & 0.167 & 0.150 & 0.135 & 0.120 & 0.107 \\
\hline 5 & $\begin{array}{l}0.24 \\
6\end{array}$ & 0.230 & 0.215 & 0.200 & 0.186 & 0.172 & 0.159 & 0.147 \\
\hline 6 & $\begin{array}{l}0.28 \\
8\end{array}$ & 0.275 & 0.263 & 0.250 & 0.238 & 0.226 & 0.214 & 0.203 \\
\hline 7 & $\begin{array}{l}0.36 \\
4\end{array}$ & 0.354 & 0.343 & 0.333 & 0.323 & 0.313 & 0.304 & 0.294 \\
\hline 8 & $\begin{array}{l}0.52 \\
1\end{array}$ & 0.514 & 0.507 & 0.500 & 0.493 & 0.486 & 0.479 & 0.472 \\
\hline 9 & $\begin{array}{l}1.00 \\
0\end{array}$ & 1.000 & 1.000 & 1.000 & 1.000 & 1.000 & 1.000 & 1.000 \\
\hline
\end{tabular}

Figure 2: Plots of hazard rate function of DMID for $\theta=10$ and for different choices of $p$.

\section{Maximum Likelihood Estimates}

Let $\mathrm{x} 1, \mathrm{x} 2, \ldots, \mathrm{xn}$ denote a random sample from this distribution, then for these fixed values the likelihood function will be

$\mathrm{L}=\frac{1}{\theta^{n p}} \cdot \prod_{i=1}^{n}\left[\left(y_{i}+1\right)^{p}-\left(y_{i}\right)^{p}\right] ; \quad \quad \mathrm{yi}=0,1,2, \ldots \ldots, \theta-1$

Or $\log \mathrm{L}=-n p \log \theta+\sum_{i=1}^{n} \log \left[\left(y_{i}+1\right)^{p}-\left(y_{i}\right)^{p}\right]$

$\frac{\partial \log L}{\partial \theta}=\frac{-n p}{\theta}=0$, has no solution for $\theta$.

We know that yi $\leq \theta-1$ or $\theta \geq \mathrm{yi}+1$ for each $\mathrm{i}$.

In particular smallest value of $\theta=$ the largest value in the sample +1 i.e. $y(n)+1$.

Thus the m.l.e. of $\theta$ is

$$
\hat{\theta}=\mathrm{y}(\mathrm{n})+1
$$

Now for m.l.e. of 'p'

$\frac{\partial \log L}{\partial p}=-n \cdot \log \theta+\sum_{i=1}^{n} \frac{\partial \log \left[\left(y_{i}+1\right)^{p}-\left(y_{i}\right)^{p}\right]}{\partial p}=0$

$-n . \log \hat{\theta}+\sum_{i=1}^{n} \frac{\left(y_{i}+1\right)^{p} \log \left(y_{i}+1\right)^{p}-\left(y_{i}\right)^{p} \log \left(y_{i}\right)^{p}}{\left(y_{i}+1\right)^{p}-\left(y_{i}\right)^{p}}=0 ;$

Solving the equation (10), obtain m.l.e. of 'p'

\section{Conclusion}

In the present paper, a discrete version of Mukherjee-Islam continuous distribution has been introduced. The various features of DMID such as cdf, reliability function and hazard rate function were also discussed with numerical illustrations.

\section{References}

Aafaq Ahmad Rather, C. Subramanian (2018):“Exponentiated Mukherjee-islam Distribution”, Journal of Statistics Applications \& Probability, Vol. 07, No. 02, pp. 357-361.

Chakraborty, S. (2015):"A new discrete distribution related to generalized gamma distribution and its properties", Communication in Statistics - Theory and Methods Vol. 44, pp. 169-1705. 
Jain, Sanjay (2015):“A Stochastic Model for Waiting Time of First Conception”, Inq. Teacher (ISSN:23483717), Vol. II, Issue I, pp. 42-46.

Javid Gani Dar, Uzma Manzoor, Essey Kebede Muluneh (2018): "Weighted Mukherjee-Islam Distribution and Its Application", International Journal of Statistics and Economics (ISSN:,0975-556X) Vol. 19, No. 02. Pp. 89-107.

Jayakumar, K.and Girish Babu, M. (2017):"Discrete Weibull geometric distribution and its properties", Communications in Statistics - Theory and Methods, Vol. 47, No. 07, pp. 1767-1783.

Mukheerji, S. P. and Islam, A. (1983):“A Finite Range Distribution of Failures Times”, Naval Research Logistics Quarterly, Vol. 30,pp. $487-491$.

Siddiqui, S.A., Dwivedi, S., Dwivedi, P., Alam, M (2016):"Beta Exponentiated Mukherjee-islam Distribution: Mathematical Study of Different Properties, Global J. of Pure and Appl. Maths., (ISSN:0973-1768), Vol. 12, Vo. 01, pp. 951-964. 